\title{
Genomic resources for the North American water vole (Microtus richardsoni) and the
} montane vole (Microtus montanus)

Drew J. Duckett ${ }^{*}$, Jack Sullivan ${ }^{2}$, Stacy Pirro $^{3}$, Bryan C. Carstens ${ }^{1}$

${ }^{1}$ Department of Evolution, Ecology, and Organismal Biology. The Ohio State University. 1315

Kinnear Rd., Columbus OH, 43212

${ }^{2}$ Department of Biological Sciences, Box 443051, University of Idaho, Moscow ID, 83844-3051

$1 \quad{ }^{3}$ Iridian Genomes, Inc., 6213 Swords Way, Bethesda MD 20817

*email: duckettdj@gmail.com 


\section{Abstract}

3 Background: Voles of the genus Microtus are important research organisms, yet genomic

4 resources in the genus are lacking. Providing such resources would benefit future studies of

5 immunology, phylogeography, cryptic diversity, and more. Findings: We sequenced and

6 assembled nuclear genomes from two subspecies of water vole (Microtus richardsoni) and from

7 the montane vole (Microtus montanus). The water vole genomes were sequenced with Illumina

8 and 10X Chromium plus Illumina sequencing, resulting in assemblies with $\sim 1,600,000$ and

$9 \sim 30,000$ scaffolds respectively. The montane vole was assembled into $\sim 13,000$ scaffolds using

10 Illumina sequencing also. In addition to the nuclear assemblies, mitochondrial genome

11 assemblies were also performed for both species. We conducted a structural and functional

12 annotation for the best water vole nuclear genome, which resulted in 24,500 annotated genes,

13 with $83 \%$ of these receiving functional annotations. Finally, we find that assembly quality

14 statistics for our nuclear assemblies fall within the range of genomes previously published in the 15 genus Microtus, making the water vole and montane vole genomes useful additions to currently

16 available genomic resources.

17

18 Keywords: genome assembly; genome annotation; mitochondrial genome; 10X Chromium;

19 Illumina sequencing 


\section{Context}

The genus Microtus consists of 62 species of voles distributed throughout North America,

27 Europe, and Asia [1]. Microtus is believed to have experienced rapid speciation and

28 diversification, with all speciation events occurring within the past four million years [2, 3], and

29 it has been suggested that some nominal species, such as M. pennsylvanicus, contain cryptic

30 diversity [4]. Microtus has been an important model system across multiple biological

31 disciplines, including studies of adaptation (e.g., [5]), infectious disease (e.g., [6]), parental care

32 (e.g., [7]), and population dynamics (reviewed in [8]). The rapid radiation of Microtus voles has

33 hindered systematic classification, leading to multiple taxonomic revisions and conflicting

34 phylogenetic analyses $[1,9,10]$. Consequently, both species boundaries and relationships among

35 species are difficult to infer. Genomic resources within Microtus will help resolve these

36 questions, and resources have steadily increased in recent years. Currently, four Microtus species

37 have assembled genomes on GenBank, two European species (M. agrestis and M. arvalis) and

two North American species (M. ochrogaster; [11], and M. oeconomus). The present study

provides resources for two additional species: M. richardsoni and M. montanus.

The North American water vole (M. richardsoni) is adapted to a semiaquatic lifestyle, relying

41 on alpine and sub-alpine streams for creating burrows and escaping predators [12]. Like other

42 semiaquatic mammals (e.g., otters), it is likely that adaptations to this lifestyle have been driven

43 by natural selection [13-15]. Water voles are among the largest species of Microtus and are

44 known for making runways of stamped-down vegetation along streams through frequent

45 movement $[12,16]$. Unlike most other vole species, $M$. richardsoni does not appear to

46 experience regular population boom and bust cycles, although population size in the species may

47 be correlated to levels of precipitation [17]. Despite being listed as Least Concern by the IUCN 
Redlist [18], the species is listed as Critically Imperiled by the Wyoming Natural Diversity

Database due to its specific habitat requirements, which can be substantially degraded by

livestock grazing [19]. Microtus richardsoni occupies a large, disjunct distribution in the Pacific

Northwest of North America, with habitat in the Cascades Mountains and the Rocky Mountains,

M. r. macropus in the central Rocky Mountains and Wyoming, and M. r. myllodontus in Utah.

Due to the subspecific classifications and the disjunct range of the species, $M$. richardsoni has

species limits and demographic history were limited to inferences that can be derived from a address these knowledge gaps.

The montane vole (M. montanus) is partially sympatric with M. richardsoni and can be found throughout most of the water vole's range with the exception of the Canadian Rockies. However, M. montanus can be found farther south and east including areas of California, Nevada,

64 Colorado, Arizona and New Mexico [23]. The species has been divided into fifteen subspecies,

65 including M. m. canescens in the Cascades Mountains., M. m. nasus in the central Rocky

66 Mountains, and M. m. amosus in northern Utah. Notably, M. montanus does not exhibit a break

67 in its range in the Columbia Basin, likely because it is not restricted to riparian areas like $M$. richardsoni. The species as a whole is listed as Least Concern by the IUCN Redlist, but M. m. 
71 declining population size [24]. Genomic resources in M. montanus will provide a wealth of data to assess subspecies boundaries, quantify gene flow among subspecies, and aid in conservation

73 efforts of threatened subspecies.

74 The present study provides two nuclear and one mitochondrial genome assembly for $M$. richardsoni along with single nuclear and mitochondrial genome assemblies for M. montanus. Furthermore, a structural and functional annotation are performed with one of the M. richardsoni genomes to aid in future studies of adaptation. Genome-level comparisons are made between the new genome assemblies and other Microtus genome assemblies to examine differences in assembly quality and repeat content.

\section{Sequencing and Nuclear Genome Assembly}

Frozen tissue from a single M. r. arvicoloides individual collected from the southern Cascades Mountain range (JMS_292; 44.016667N, -121.750000E; [20]) was sent to Hudson Alpha

86 before the fragment is sheared for library preparation. After sequencing, these barcodes are used

87 to connect sequencing reads for a more contiguous assembly. After sequencing with a single run

88 on an Illumina HiSeqX, the resulting 150 base pair $(\mathrm{bp})$ paired-end reads were input into

89 Supernova for de novo genome assembly with --maxreads=all [26].

90 Additional tissue was obtained from a single M. r. macropus individual collected from the

91 northern Rocky Mountains (JMG_88; 46.333333N, -114.633333E; [20]). DNA was extracted

92 using a Qiagen DNeasy Blood and Tissue Kit, and the DNA was sent for library preparation and

93 sequencing by Iridian Genomes, Inc (Bethesda, MD). 150bp paired-end reads were sequenced on 
94 two runs of an Illumina HiSeqX. Genome assembly was performed using two different deBruijn 95 graph-based programs, SOAPdenovo and Discovar de novo [27, 28]. For SOAPdenovo, quality

96 trimming was performed using fastQC and Trimmomatic with settings ILLUMINACLIP:

97 2:30:10, LEADING:3, TRAILING:3, SLIDINGWINDOW:4:15, and MINLEN:36 [29, 30].

98 SOAPdenovo assemblies were performed with settings max_rd_len=150, avg_ins=300,

99 reverse_seq $=0$, asm_flags $=3$, rd_len_cutoff $=150$, rank $=1$, pair_num_cutoff $=3$, and map_len $=32$.

100 SOAPdenovo was run with kmer values of 63, 89, 95, and 101 based on analysis of optimal kmer

101 values in kmerGenie [31]. Raw reads were used as input for de novo genome assembly with

102 Discovar as recommended in the program documentation.

103 In an attempt to provide the most contiguous assembly for M. richardsoni, a hybrid assembly

104 was performed using the ARCS+LINKS pipeline $[32,33]$. The ARCS+LINKS pipeline uses

105 barcoding information from the 10X Chromium reads to scaffold the contigs from a separate

106 genome assembly. Barcoded reads from M. r. arvicoloides were mapped to the M. r. macropus

107 Discovar assembly with bwa mem [34] before converting the mapped reads to BAM format and

108 sorting with SAMTools [35]. ARCS and LINKS were then run with settings $-\mathrm{s} 98-\mathrm{c} 5-10-\mathrm{Z}$

$109500-\mathrm{d} 0-\mathrm{r} 0.05-\mathrm{m} 50-10000-\mathrm{e} 30000$ and $-\mathrm{d} 4000-\mathrm{k} 20-15-\mathrm{t} 2-\mathrm{a} 0.3-\mathrm{o} 0-\mathrm{a} 0.3-\mathrm{z} 500$

110 respectively.

111 As part of a separate project, a single M. montanus individual from Utah

112 (UMNH:Mamm:30891; 38.19381N, -111.5824E) was misidentified as M. richardsoni. DNA was

113 extracted from the sample using a Qiagen DNeasy Blood and Tissue Kit before being sent to the

114 University of California Davis Genome Center for library preparation and sequencing. Paired-

115 end 150bp sequences were collected with a single shared run on an Illumina NovaSeq. Species

116 identity was confirmed using the Barcode of Life Database (BOLD; [36]). Reads were checked 
117 and trimmed for quality with fastQC and Trimmomatic as above before mapping reads to the

118 mitochondrial cytochrome oxidase I (COI) sequence of M. r. macropus [37] using bwa mem.

119 The resulting mapped reads were converted to BAM format, sorted, and indexed with

120 SAMTools. PCR duplicates were identified and removed with Picard [38], resulting reads were

121 piled with SAMTools mpileup using base and mapping quality scores of 30, consensus

122 sequences were generated with bcftools [39], and consensus sequences were converted to fastq

123 format using vcfutils with a minimum depth filter of 5 and maximum depth filter of 10000 [35].

124 The resulting sequence was input into BOLD. Due to the low sequencing coverage, de novo

125 genome assembly was not appropriate for M. montanus. To provide a preliminary genome

126 sequence, a reference-guided genome assembly was performed with RaGOO [40]. Raw reads

127 were input into Discovar to generate an initial genome assembly, misassembly correction was

128 performed with RaGOO using reads trimmed with the same settings as the M.r. macropus reads,

129 and RaGOO was then used to scaffold the Discovar contigs onto the $M$. $r$. arvicoloides assembly,

130 which is more closely related to M. montanus than the other available Microtus genome

131 assemblies [3]. Since M. montanus has less than half the chromosomes of $M$. richardsoni $(2 \mathrm{n}=$

132 22-24 in montanus versus 56 in richardsoni [41]), the possibility of structural errors in the $M$.

133 montanus assembly was examined by calculating the percentage of reads that mapped back to the

134 assembly using bwa mem and bamtools [42].

135 The final assemblies were submitted to GenBank [43], where screening was performed to

136 identify any contamination, and contaminated scaffolds were removed. All assemblies were

137 evaluated with QUAST [44], bbmap [45], custom Python scripts

138 (https://github.com/djlduckett/Genome_Resources/), and BUSCO using the Euarchontoglires

139 reference set [46]. After comparing assembly statistics from the different assemblies of M. $r$. 
macropus, the Discovar assembly was selected as best because it had less fragmentation, higher

141 N50 and L50, and a higher BUSCO score than the SOAPdenovo assemblies (Table 1). Genome

142 sequencing of M. r. arvicoloides produced over 800 million (M) reads and 47x genome

143 sequencing coverage. The final genome assembly consisted of $\sim 32$ thousand $(\mathrm{K})$ scaffolds with

144 an N50 of 2.3 megabase pairs $(\mathrm{Mb}), 1.3 \%$ missing data $(\mathrm{N})$, and a BUSCO score of $85.8 \%$.

145 Supernova estimated the length of the genome assembled to be $\sim 2.4 \mathrm{~Gb}$ and the total genome size

146 to be $\sim 2.6$ gigabase pairs (Gb). Microtus richardsoni macropus sequencing produced over 600M

147 reads and $35 \mathrm{x}$ coverage. Genome assembly with Discovar resulted in $\sim 1.6 \mathrm{M}$ scaffolds with an

148 N50 of 16 kilobase pairs $(\mathrm{Kb}), 0.06 \% \mathrm{Ns}$, and a BUSCO score of $54.5 \%$. Given that there are

149 many programs to perform de novo genome assembly from short reads, it is possible that another

150 program would have produced a more contiguous $M . r$. macropus assembly, but previous studies

151 have shown Discovar performs well compared to other programs $[47,48]$. The hybrid assembly

152 produced with the ARCS+LINKS pipeline had $\sim 1.6 \mathrm{M}$ scaffolds, an N50 of 38Kb, 0.09\% Ns, and

153 a BUSCO score of $59.8 \%$. Because of the poor quality of the hybrid assembly, it was not used

154 for further analyses, and the M. richardsoni subspecies assemblies were kept separate. It seems

155 likely that the high fragmentation of the hybrid assembly is due to the fragmentation of the

156 Discovar input assembly. Published results with this hybrid pipeline often include a much higher

157 sequencing coverage of the input contigs to produce a better starting point for the pipeline.

158 Therefore, additional Illumina sequencing with M.r. macropus in the future could substantially

159 improve the hybrid assembly. $108 \mathrm{M}$ reads (13x coverage) were used to produce the preliminary

160 M. montanus genome, resulting in $\sim 13 \mathrm{~K}$ scaffolds, an N50 of $\sim 3.1 \mathrm{Mb}, 8.8 \% \mathrm{Ns}$, and a BUSCO

161 score of $82.6 \%$. Additionally, $89.3 \%$ of reads mapped back to the M. montanus assembly.

162

163 
Table 1

\begin{tabular}{c|ccccc} 
& Discovar & SOAPdenovo & SOAPdenovo & SOAPdenovo & SOAPdenovo \\
\hline Kmer & NA & 63 & 89 & 95 & 101 \\
Length & $2.54 \mathrm{~Gb}$ & $2.72 \mathrm{~Gb}$ & $2.88 \mathrm{~Gb}$ & $2.89 \mathrm{~Gb}$ & $3.21 \mathrm{~Gb}$ \\
Scaffolds & $1.6 \mathrm{M}$ & $4.1 \mathrm{M}$ & $4.0 \mathrm{M}$ & $4.1 \mathrm{M}$ & $6.7 \mathrm{M}$ \\
$\begin{array}{c}\text { Max } \\
\text { Scaffold }\end{array}$ & $264 \mathrm{~Kb}$ & $186 \mathrm{~Kb}$ & $146 \mathrm{~Kb}$ & $174 \mathrm{~Kb}$ & $139 \mathrm{~Kb}$ \\
N50 & $16.1 \mathrm{~Kb}$ & $4.5 \mathrm{~Kb}$ & $3.4 \mathrm{~Kb}$ & $3.4 \mathrm{~Kb}$ & $1.5 \mathrm{~Kb}$ \\
L50 & $35.7 \mathrm{~K}$ & $117 \mathrm{~K}$ & $156 \mathrm{~K}$ & $163 \mathrm{~K}$ & $371 \mathrm{~K}$ \\
BUSCO & $54.5 \%$ & $38.1 \%$ & $37.1 \%$ & $35.9 \%$ & $25.9 \%$ \\
$\%$ N & 0.06 & 1.45 & 0.99 & 0.94 & 0.90 \\
$\%$ GC & 42.13 & 41.92 & 41.91 & 41.92 & 41.98
\end{tabular}

\section{Mitochondrial Genomes}

168 The complete mitochondrial genomes of M. r. arvicoloides and M. montanus were assembled

169 using the genomic sequencing reads. The mitochondrial genomes were assembled by both

170 mapping reads to a reference mitochondrial genome and using the reference-guided assembly

171 program Novoplasty [49]. For the mapping assembly, reads were mapped to the M. r. macropus

172 mitochondrial genome, using the same steps as the M. montanus BOLD analysis. The

173 mitochondrial assemblies were $16,285 \mathrm{bp}$ and $16,268 \mathrm{bp}$ in length with an average depth of

174 coverage of $7886 \mathrm{x}$ and $6805 \mathrm{x}$ for $M$. r. arvicoloides and M. montanus respectively. Reference

175 guided mitochondrial assemblies with Novoplasty used the M. r. macropus mitochondrial

176 genome as the reference along with settings Genome Range=12000-22000, K-mer=33, Read 
177 Length $=150$, and Insert size $=400$. Because the M. r. arvicoloides dataset contained many reads,

$17825 \%$ of reads were subsampled to use for assembly, as suggested in the program documentation.

179 The assemblies for M. r. arvicoloides and M. montanus were 16,298bp and 16,319bp in length

180 with average depths of coverage of 5131x and 14,713x respectively. To compare mitochondrial

181 assemblies between methods, the assemblies were aligned using the MUSCLE plugin in

182 Geneious v. R9 with eight iterations and an open gap score of -1 [50, 51]. This comparison

183 showed the Novoplasty assemblies contained multiple insertions compared to the mapped

184 assemblies and the reference mitochondrial genome. These insertions were up to 13bp long in

185 multiple genes, including trnT, trnK, and ATP8. Comparison to other Microtus mitochondrial

186 genomes (M. ochrogaster; NC_027945.1 and M. fortis; NC_015243.1) showed that the

187 Novoplasty assemblies were the only mitochondrial assemblies to exhibit these insertions.

188 Therefore, the mapping assemblies were used for further analyses. The mapping assemblies for

189 both species included ambiguous bases, which were much more frequent for M. montanus than

190 M. r. arvicoloides. These may be the result of using the mitochondrial genome of a different

191 subspecies (for M. r. arvicoloides) or species (for M. montanus) for mapping the reads.

192 Additionally, the presence of nuclear DNA of mitochondrial origin (NUMTs; [52, 53]) may have

193 influenced these results. If mitochondrial segments have been incorporated into the nuclear

194 genomes and subsequent mutations have occurred, both nuclear and mitochondrial sequences

195 could be mapped to the same mitochondrial region during assembly and result in the ambiguous

196 bases observed here. It is likely that NUMTs are present, as they have been documented in other

197 species of Microtus [54-56]. Both mitochondrial genomes were annotated using MITOS [57].

198 The annotations each consisted of 22 tRNA genes, 2 rRNA genes, and 13 protein coding genes. 


\section{Microtus Genome Assembly Comparison}

201 The available Microtus genome assemblies, M. agrestis (GCA_902806755.1), M. arvalis

202 (GCA_007455615.1), M. ochrogaster (GCA_000317375.1), and M. oeconomus

203 (GCA_007455595.1), were downloaded from GenBank. Assembly summary statistics were

204 calculated using QUAST, bbmap, and custom Python scripts

205 (https://github.com/djlduckett/Genome_Resources/). To compare repeat content among all

206 genomes, including the three produced by the current study, repeats were first identified de novo

207 using RepeatModeler [58]. RepeatMasker was then used to further identify repeats using a

208 combined repeat library that included the repeats identified from RepeatModeler and those from

209 the RepeatMasker Rodentia database [59]. The percentage of the genome consisting of each type

210 of repeat element was extracted from the RepeatMasker log file for each genome assembly.

211 All genome assemblies used some form of Illumina sequencing (Table 2), although assembly

212 continuity varied greatly among assemblies from 1366 scaffolds in M. agrestis to $1.6 \mathrm{M}$ scaffolds

213 in M. r. macropus. Genome coverage was similarly varied, from 13x in M. montanus to $35 \mathrm{x}$ in

214 M. r. macropus to $77 \mathrm{x}$ in M. arvalis and M. oeconomus. The percent of repetitive regions ranged

215 from $31.7 \%$ in M. montanus to $44.1 \%$ in M. arvalis (Figure 1), and repeat content did not appear

216 to be associated with phylogenetic relatedness as repeats between the two subspecies of $M$.

217 richardsoni were not more similar to each other than to other Microtus species. However, it is

218 possible that the repeat content is affected by the continuity of the genome assemblies, and

219 further research is needed to confirm this relationship. 
Table 2

\begin{tabular}{|c|c|c|c|c|c|c|c|}
\hline Species & M. agrestis & M. arvalis & M. montanus* & M. ochrogaster & M. oеconomus & $\begin{array}{c}\text { M. } r . \\
\text { arvicoloides* }\end{array}$ & M. r. macropus* \\
\hline Distribution & Europe & Europe & North America & North America & North America & North America & North America \\
\hline Year & 2020 & 2019 & 2020 & 2012 & 2019 & 2020 & 2020 \\
\hline $\begin{array}{l}\text { Accession } \\
\text { (GCA_) }\end{array}$ & 902806775.1 & 7455615.1 & $\operatorname{xxxxxxxxxxx}$ & 317375.1 & 7455595.1 & xxxxxxxxxxx & $\operatorname{xxxxxxxxxxx}$ \\
\hline Sequencing & $\begin{array}{l}\text { 10X Chromium + } \\
\text { Illumina }\end{array}$ & Illumina & Illumina & Illumina & Illumina & $\begin{array}{l}\text { 10X Chromium }+ \\
\text { Illumina }\end{array}$ & Illumina \\
\hline Assembler & Supernova & Discovar & RaGOO & ALLPATHS & Discovar & Supernova & Discovar \\
\hline Length & $2.03 \mathrm{~Gb}$ & $2.62 \mathrm{~Gb}$ & $2.34 \mathrm{~Gb}$ & $2.29 \mathrm{~Gb}$ & $2.31 \mathrm{~Gb}$ & $2.36 \mathrm{~Gb}$ & $2.54 \mathrm{~Gb}$ \\
\hline Coverage & 50 & 77 & 13 & 94 & 77 & 47 & 35 \\
\hline \# Scaffolds & 1,366 & $1,081,432$ & 12,962 & 6,341 & 562,436 & 31,632 & $1,648,927$ \\
\hline $\begin{array}{l}\text { Longest } \\
\text { Scaffold }\end{array}$ & $56.96 \mathrm{Mb}$ & $0.80 \mathrm{Mb}$ & $748.72 \mathrm{Mb}$ & $126.73 \mathrm{Mb}$ & $0.93 \mathrm{Mb}$ & $16.00 \mathrm{Mb}$ & $0.26 \mathrm{Mb}$ \\
\hline N50 & $13.35 \mathrm{Mb}$ & $0.53 \mathrm{Mb}$ & $3.08 \mathrm{Mb}$ & $61.81 \mathrm{Mb}$ & $0.11 \mathrm{Mb}$ & $2.30 \mathrm{Mb}$ & $0.02 \mathrm{Mb}$ \\
\hline L50 & 45 & 11,870 & 91 & 14 & 5,556 & 278 & 35,660 \\
\hline$\% \mathrm{~N}$ & 2.87 & 0.07 & 8.81 & 8 & 0.12 & 1.29 & 0.06 \\
\hline$\% G C$ & 42.33 & 41.71 & 42.38 & 42.25 & 42.18 & 42.21 & 42.13 \\
\hline
\end{tabular}

221 Genome assembly comparison among Microtus species. Assemblies with a * were produced by the present study. Note: in-depth methods for M. agrestis are not

222 available, and it is possible that the assembly includes additional sequencing and/or methods. 


\section{Genome Annotation}

The M. r. arvicoloides genome assembly was annotated with the MAKER pipeline [60],

225 loosely following the tutorial provided by Daren Card (https://gist.github.com/darencard

226 /bb1001ac1532dd4225b030cf0cd61ce2). Briefly, the pipeline consists of masking repeats

227 followed by multiple rounds of annotation with both evidence-based and ab-initio gene models.

228 Repeats were identified as described above. Complex repeats were then extracted from

229 RepeatMasker results using grep with keywords "Satellite" and "rich". Within Maker, the

230 model_org argument was set to "simple" so Maker would soft mask simple repeats, and the

231 RepeatMasker results were provided to hard mask complex repeats. Evidence-based gene

232 discovery used protein and mRNA sequences from the previous genome annotation of $M$.

233 ochrogaster (GCF_000317375.1) as well as an additional RNASeq assembly from $M$.

234 pennsylvanicus (GSM3499528; [61]). Hidden Markov models (HMMs) for ab-initio gene

235 prediction were trained using both SNAP and Augustus [62, 63]. With SNAP, gene models

236 identified by MAKER were filtered using an Annotation Edit Distance (AED) of 0.5 and an

237 amino acid length of 50. After validating these models with SNAP's Fathom utility, removing

238 likely errors, and including $1000 \mathrm{bp}$ surrounding each training sequence, the training sequences

239 were passed to the hmm-assembler script. For Augustus, training sequences plus 1000bp on each

240 side were obtained from the first round of MAKER mRNA annotations. Augustus was used to

241 train the HMM using the --long option in BUSCO and the Euarchontoglires reference set.

242 MAKER was then run again with the previously annotated gene models and the HMM models

243 from SNAP and Augustus. After the initial MAKER run, two cycles of ab-initio gene prediction

244 and annotation with MAKER were performed. To prevent overfitting, results were compared

245 after each round of MAKER. Because the increase in AED score was minimal between the first 
and second rounds of ab-initio gene prediction, further analysis was conducted on the results

247 after the first round only. This round annotated $\sim 24 \mathrm{~K}$ genes with a mean gene length of $7445 \mathrm{bp}$

248 (Table 3), which is within the range found in previous studies of $M$. ochrogaster $(22,427$ genes;

249 GCF_000317375.1) and Arvicola amphibious (25,136 genes; GCF_903992535.1). Of these

250 annotations all occurred on scaffolds greater than $1 \mathrm{~Kb}$ in length and $97 \%$ occurred on scaffolds

251 greater than $10 \mathrm{~Kb}$ in length.

252 Functional annotation of the M. r. arvicoloides genome was performed using GOfeat, an

253 online functional annotation tool that uses multiple protein databases including UniProt,

254 InterPro, and Pfam [64-67]. An input file for GOfeat was generated by supplying the genome

255 assembly FASTA file and the MAKER General Feature Format (GFF3) file to the Python

256 package gffread [68]. GOfeat annotated 83.49\% of genes. Biological Processes accounted for

$25742.46 \%$ of annotations, Cellular Components accounted for 30.29\%, and Molecular Functions

258 comprised $27.25 \%$. The most frequent gene ontology $(\mathrm{GO})$ terms were positive regulation of

259 transcription by RNA polymerase II, negative regulation of transcription by RNA polymerase II,

260 and DNA-templated regulation of transcription for Biological Processes, cytoplasm and plasma

261 membrane for Cellular Components, and metal ion binding and calcium ion binding for

262 Molecular Functions. 
Table 3

Before Gene Modeling

Gene Modeling Round 1 Gene Modeling Round 2

\begin{tabular}{lccc}
\hline Genes & 20,945 & 24,548 & 23,811 \\
Exons & 139,845 & 192,974 & 179,225 \\
mRNA & 20,945 & 24,548 & 23,811 \\
tRNA & - & 24,504 & 24,539 \\
$\mathbf{5}$, UTR & - & 1,229 & 1,180 \\
3' UTR & - & 503 & 642 \\
Mean Gene Length & - & 7,445 & 7,132 \\
AED $<\mathbf{0 . 5 0}$ & 0.993 & 0.881 & 0.888 \\
AED $<\mathbf{0 . 2 5}$ & 0.672 & 0.543 & 0.520 \\
BUSCO (Complete) & - & $67.7 \%$ & $70.5 \%$
\end{tabular}

271 Structural annotation summary after each round of MAKER. UTR: untranslated region; AED: annotation edit

272 distance. Values with dashes were not analyzed prior to gene modeling with SNAP and Augustus.

\section{Conclusion}

275 The current study details the assembly and annotation of three nuclear and two mitochondrial

276 genomes. Compared to previously published nuclear genomes, the M. r. arvicoloides and $M$.

277 montanus genomes are of high quality as evidenced by the low number of scaffolds, high

278 N50/L50 values, and high BUSCO scores. While not as complete as the other Microtus genomes,

279 the nuclear genome of M. r. macropus will still be useful for mapping low coverage reads or

280 reduced representation sequencing data. Furthermore, the mitochondrial genomes contributed

281 here add to a growing number for the genus Microtus and reinforce earlier suggestions that high-

282 quality mitochondrial genomes can be obtained as byproducts of nuclear sequencing (e.g., [69,

283 70]). Overall, the data presented serve as an example that even though they do not include 
284 chromosomal information, high-quality draft genomes can be produced from widely available

285 and very cost-effective methods like the 10X Chromium protocol. These references can aid a

286 variety of studies including those examining genus and species adaptation [71, 72],

287 phylogenetics [10], phylogeography [22, 73], and disease dynamics [6, 74]. However, some

288 activities, like exploring changes to chromosome structure, will not be possible due to the

289 fragmentation and lack of chromosomal mapping for these assemblies. Finally, the M. $r$.

290 macropus and $M$. montanus sequencing data and preliminary assemblies will serve as the

291 building blocks of more accurate reference genomes in the future.

292

293 Availability of Supporting Data and Materials

294 Raw sequences, nuclear assemblies, and mitochondrial assemblies are available from GenBank 295 under BioProjects PRJNA673719, PRJNA509068, and PRJNA673873 for M. r. arvicoloides, M.

296 r. macropus, and M. montanus respectively. The custom python script used to calculate genome

297 assembly summary information is available on GitHub

298 (https://github.com/djlduckett/Genome_Resources/). Full BUSCO tables, structural annotation

299 gff files, functional annotation tables, and repeat libraries are available in the GigaScience data

300 repository (http://gigadb.org/).

301

302

303

304

305

306 


\section{Figures:}

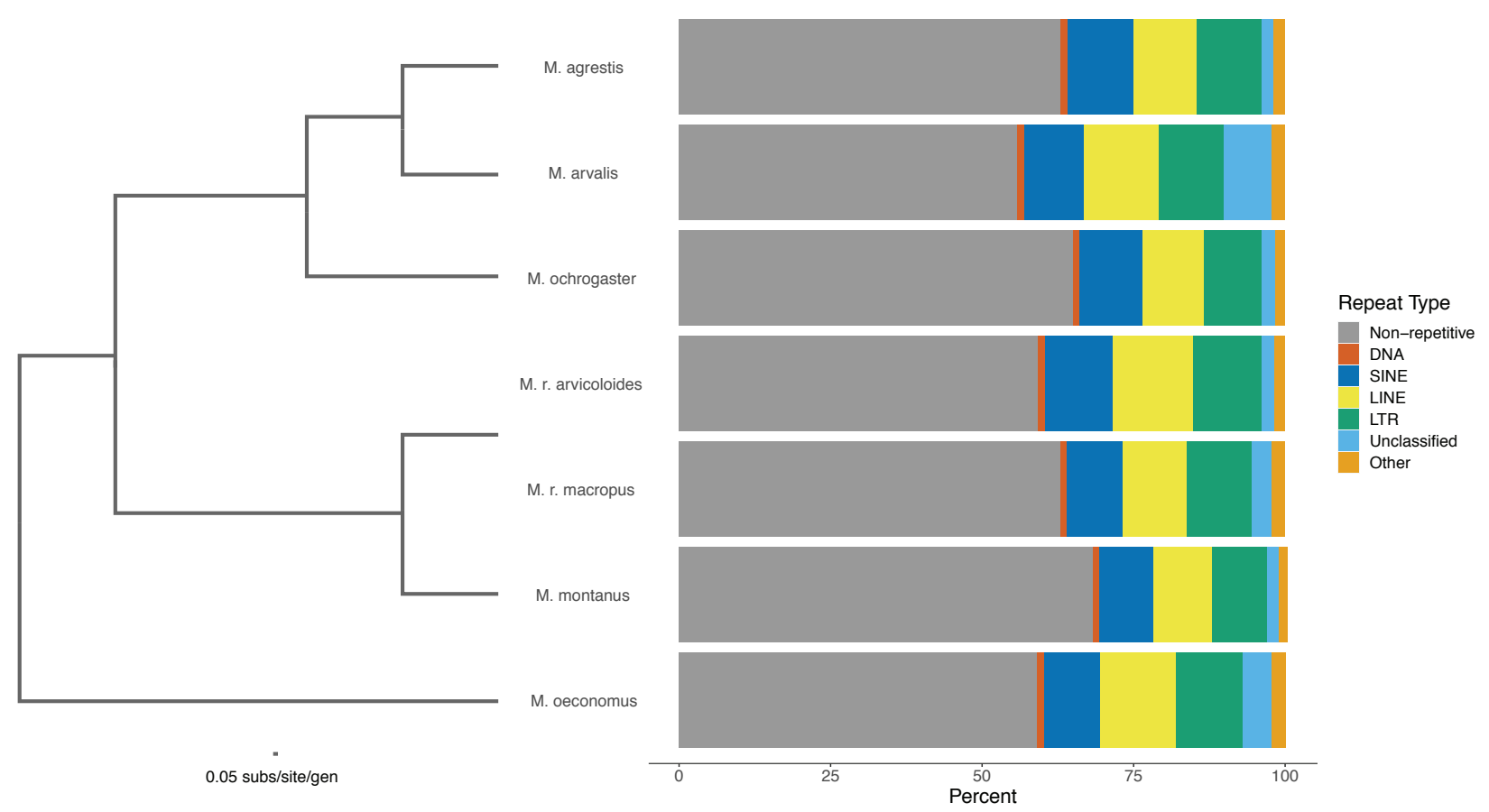

Figure 1: Repeat Content among Microtus genomes. SINE: short interspersed nuclear element; LINE: long

310 interspersed nuclear element; LTR: long terminal repeat; Other consists of small RNA, satellite, simple, and low

311 complexity repeats. The phylogeny displayed was recreated from [3] by pruning unincluded species from the data

312 alignment and rerunning RAxML [75] with the same settings used in the original analysis.

\section{Abbreviations}

315 BOLD: Barcode of Life Database; bp: base pair; COI: cytochrome oxidase I; M: million; K:

316 thousand; Gb: gigabase pairs; Mb: megabase pairs; Kb: kilobase pairs; SINE: short interspersed

317 nuclear element; LINE: long interspersed nuclear element; LTR: long terminal repeat; AED:

318 annotation edit distance; HMM: hidden Markov model; NUMT: nuclear DNA of mitochondrial

319 origin 


\section{Competing Interests}

323 SP is the director of Iridian Genomes, Inc.

325 Funding

326 Sequencing was funded by Iridian Genomes, Inc., as well as the National Science Foundation

327 (DEB-1457519). Salary support for DD was provided by The Ohio State University and the

328 National Science Foundation (DBI-1945347).

\section{Author Contributions}

331 DD, JS, and BC conceived the study. JS, SP, and BC provided funding for sequencing. DD

332 performed DNA extractions, assembled genomes, and annotated genomes with input from SP.

333 DD and BC wrote the manuscript with input from JS and SP. DD and SP submitted the resources

334 to GenBank.

336 Acknowledgements

337 We thank Jeffrey Good and Eric Rickart/Utah Museum of Natural History for tissue samples,

338 Michael Broe for advice with genome assembly and annotation, and the Ohio Supercomputer

339 Center (OSC) for computational resources. 


\section{References}

1. Wilson DE, Reeder DM, editors. Mammal species of the world: a taxonomic and geographic reference. JHU Press; 2005.

2. Reig OA. Karyotypic repatterning as one triggering factor in cases of explosive speciation. In:

Fontdevila A, editor. Evolutionary biology of transient unstable populations. Springer, Berlin, Heidelberg; 1989 p. 246-289.

3. Steppan SJ, Schenk JJ. Muroid rodent phylogenetics: 900-species tree reveals increasing diversification rates. PloS one. 2017;12:8.

4. Jackson DJ, Cook JA. A precarious future for distinctive peripheral populations of meadow voles (Microtus pennsylvanicus). Journal of Mammalogy. 2020;101:1:36-51.

5. Monarca RI, Speakman JR, da Luz Mathias M. Energetics and thermal adaptation in semifossorial pine-voles Microtus lusitanicus and Microtus duodecimcostatus. Journal of Comparative Physiology B. 2019;189:2:309-18.

6. Wanelik KM, Begon M, Birtles RJ, Bradley JE, Friberg IM, Jackson JA, Taylor CH, Thomason AG, Turner AK, Paterson S. A candidate tolerance gene identified in a natural population of field voles (Microtus agrestis). Molecular ecology. 2018;27:4:1044-52.

7. Seelke AM, Perkeybile AM, Grunewald R, Bales KL, Krubitzer LA. Individual differences in cortical connections of somatosensory cortex are associated with parental rearing style in prairie voles (Microtus ochrogaster). Journal of Comparative Neurology. 2016;524:3:564-77. Mammal Review. 2019;49:3:226-39.

88. 
10. Barbosa S, Paupério J, Pavlova SV, Alves PC, Searle JB. The Microtus voles: Resolving the phylogeny of one of the most speciose mammalian genera using genomics. Molecular phylogenetics and evolution. 2018;125:85-92.

11. McGraw LA, Davis JK, Lowman JJ, ten Hallers BF, Koriabine M, Young LJ, De Jong PJ, Rudd MK, Thomas JW. Development of genomic resources for the prairie vole (Microtus ochrogaster): construction of a BAC library and vole-mouse comparative cytogenetic map. BMC genomics. 2010;11:1:1-8.

12. Klaus M, Beauvais GP. Water Vole (Microtus richardsoni): A Technical Conservation Assessment. Prepared for USDA Forest Service, Rocky Mountain Region, Species Conservation Project. 2004.

377 13. Stein BR. Bone density and adaptation in semiaquatic mammals. Journal of mammalogy. 1989;70:3:467-76.

14. Dunstone N, Gorman ML, editors. Behaviour and ecology of riparian mammals. Cambridge University Press; 2007. evolution. 2019;36:12:2631-55.

16. Ludwig DR. Microtus richardsoni. Mammalian Species. 1984;223:1-6. Beartooth Mountains of Montana and Wyoming, USA. Arctic, Antarctic, and Alpine Research. 1999;31:3:278-82.

18. Cassola, F. Microtus richardsoni. The IUCN Red List of Threatened Species 2016.

19. Wyoming Natural Diversity Database. University of Wyoming. 2020. https://wyndd.org/species_list/. 
20. Cartens BC, Brunsfeld SJ, Demboski JR, Good JM, Sullivan J. Investigating the evolutionary history of the Pacific Northwest mesic forest ecosystem: hypothesis testing within a comparative phylogeographic framework. Evolution. 2005;59:8:1639-52.

21. Carstens BC, Richards CL. Integrating coalescent and ecological niche modeling in comparative phylogeography. Evolution: International Journal of Organic Evolution. 2007;61:6:1439-54.

22. Espíndola A, Ruffley M, Smith ML, Carstens BC, Tank DC, Sullivan J. Identifying cryptic diversity with predictive phylogeography. Proceedings of the Royal Society B: Biological Sciences. 2016;283:1841:20161529.

23. Sera WE, Early CN. Microtus montanus. Mammalian Species. 2003;716:1-10.

24. Cassola, F. Microtus montanus. The IUCN Red List of Threatened Species 2016. cancer genomes with high-throughput linked-read sequencing. Nature biotechnology. 2016;34:3:303-11.

26. Weisenfeld NI, Kumar V, Shah P, Church DM, Jaffe DB. Direct determination of diploid genome sequences. Genome research. 2017;27:5:757-67. 2014;46:12:1350. 
30. Bolger AM, Lohse M, Usadel B. Trimmomatic: a flexible trimmer for Illumina sequence data. Bioinformatics. 2014;30:15:2114-20.

31. Chikhi R, Medvedev P. Informed and automated k-mer size selection for genome assembly. Bioinformatics. 2014;30:1:31-7.

32. Yeo S, Coombe L, Warren RL, Chu J, Birol I. ARCS: scaffolding genome drafts with linked reads. Bioinformatics. 2018;34:5:725-31.

33. Warren RL, Yang C, Vandervalk BP, Behsaz B, Lagman A, Jones SJ, Birol I. LINKS: Scalable, alignment-free scaffolding of draft genomes with long reads. GigaScience. 2015;4:1:s13742-015.

34. Li H, Durbin R. Fast and accurate long-read alignment with Burrows-Wheeler transform. Bioinformatics. 2010;26:5:589-95. sequence alignment/map format and SAMtools. Bioinformatics. 2009;25:16:2078-9. org). Molecular ecology notes. 2007;7:3:355-64.

37. Alqahtani F, Duckett D, Pirro S, Mandoiu II. Complete mitochondrial genome of the water vole, Microtus richardsoni (Cricetidae, Rodentia). Mitochondrial DNA Part B. 2020;5:3:2498-9.Broad Institute. Picard tools. 2016. http://github.com/broadinstitute/picard.

38. Li H. A statistical framework for SNP calling, mutation discovery, association mapping and population genetical parameter estimation from sequencing data. Bioinformatics. 2011;27:21:2987-93.

39. Alonge M, Soyk S, Ramakrishnan S, Wang X, Goodwin S, Sedlazeck FJ, Lippman ZB, Schatz MC. RaGOO: fast and accurate reference-guided scaffolding of draft genomes. Genome biology. 2019;20:1:1-7. 
40. Modi WS. Phylogenetic analyses of chromosomal banding patterns among the Nearctic Arvicolidae (Mammalia: Rodentia). Systematic Zoology. 1987;36:2:109-36. for analyzing and managing BAM files. Bioinformatics. 2011;27:12:1691-2.

42. Benson DA, Cavanaugh M, Clark K, Karsch-Mizrachi I, Lipman DJ, Ostell J, Sayers EW. GenBank. Nucleic acids research. 2012;41:D1:D36-42.

43. Gurevich A, Saveliev V, Vyahhi N, Tesler G. QUAST: quality assessment tool for genome assemblies. Bioinformatics. 2013;29:8:1072-5. Lab.(LBNL), Berkeley, CA (United States); 2014. https://jgi.doe.gov/data-and-tools/bbtools/. assembly and annotation completeness with single-copy orthologs. Bioinformatics. 2015;31:19:3210-2.

47. Fernandez-Silva I, Henderson JB, Rocha LA, Simison WB. Whole-genome assembly of the coral

46. Mikheenko A, Prjibelski A, Saveliev V, Antipov D, Gurevich A. Versatile genome assembly whole genome data. Nucleic acids research. 2017;45:4:e18-.

49. Kearse M, Moir R, Wilson A, Stones-Havas S, Cheung M, Sturrock S, Buxton S, Cooper A, software platform for the organization and analysis of sequence data. Bioinformatics. 2012;28:12:1647-9. 
50. Edgar RC. MUSCLE: multiple sequence alignment with high accuracy and high throughput. Nucleic acids research. 2004;32:5:1792-7.

51. Lopez JV, Yuhki N, Masuda R, Modi W, O'Brien SJ. Numt, a recent transfer and tandem amplification of mitochondrial DNA to the nuclear genome of the domestic cat. Journal of molecular evolution. 1994;39:2:174-90.

52. Hazkani-Covo E, Zeller RM, Martin W. Molecular poltergeists: mitochondrial DNA copies (nuMountains) in sequenced nuclear genomes. PLoS Genet. 2010;6:2:e1000834.

53. Triant DA, DeWoody JA. Extensive mitochondrial DNA transfer in a rapidly evolving rodent has been mediated by independent insertion events and by duplications. Gene. 2007;401:1-2:61-70.

54. Triant DA, DeWoody JA. Molecular analyses of mitochondrial pseudogenes within the nuclear genome of arvicoline rodents. Genetica. 2008;132:1:21-33.

55. Triant DA, DeWoody JA. Demography and phylogenetic utility of numt pseudogenes in the Southern Red-Backed Vole (Myodes gapperi). Journal of mammalogy. 2009;90:3:561-70. PF. MITOS: improved de novo metazoan mitochondrial genome annotation. Molecular

59. Cantarel BL, Korf I, Robb SM, Parra G, Ross E, Moore B, Holt C, Alvarado AS, Yandell M. MAKER: phylogenetics and evolution. 2013;69:2:313-9.

57. Smit AF, Hubley R. RepeatModeler Open-1.0. 2008. http://www.repeatmasker.org .

58. Smit AF, Hubley R, Green P. RepeatMasker Open-4.0. 2013. http://www.repeatmasker.org .

60. Young RL, Ferkin MH, Ockendon-Powell NF, Orr VN, Phelps SM, Pogány Á, Richards-Zawacki CL, Summers K, Székely T, Trainor BC, Urrutia AO. Conserved transcriptomic profiles underpin 
monogamy across vertebrates. Proceedings of the National Academy of Sciences. 2019;116:4:1331-6.

61. Korf I. Gene finding in novel genomes. BMC bioinformatics. 2004;5:1:59.

62. Stanke M, Waack S. Gene prediction with a hidden Markov model and a new intron submodel. Bioinformatics. 2003;19:ii215-25.

63. Araujo FA, Barh D, Silva A, Guimarães L, Ramos RT. GO FEAT: a rapid web-based functional annotation tool for genomic and transcriptomic data. Scientific reports. 2018;8:1:1-4.

64. UniProt Consortium. UniProt: a hub for protein information. Nucleic acids research. 2015;43:D1:D204-12.

65. Hunter S, Apweiler R, Attwood TK, Bairoch A, Bateman A, Binns D, Bork P, Das U, Daugherty L, Duquenne L, Finn RD. InterPro: the integrative protein signature database. Nucleic acids research. 2009;37:D211-5. 2004;32:D138-41.

67. Pertea G, Pertea M. GFF Utilities: GffRead and GffCompare. F1000Research. 2020;9.

68. Voigt O, Erpenbeck D, Wörheide G. A fragmented metazoan organellar genome: the two mitochondrial chromosomes of Hydra magnipapillata. BMC genomics. 2008;9:1:350. 90.

70. Fink S, Excoffier L, Heckel G. Mitochondrial gene diversity in the common vole Microtus arvalis shaped by historical divergence and local adaptations. Molecular Ecology. 2004;13:11:3501-14. 
71. Fischer MC, Foll M, Excoffier L, Heckel G. Enhanced AFLP genome scans detect local adaptation in high-altitude populations of a small rodent (Microtus arvalis). Molecular Ecology. 2011;20:7:1450-62.

72. Frey JK. Genetics of allopatric populations of the montane vole (Microtus montanus) and Mogollon vole (Microtus mogollonensis) in the American Southwest. Western North American Naturalist. 2009;69:2:215-22.

512 73. Tołkacz K, Alsarraf M, Kowalec M, Dwużnik D, Grzybek M, Behnke JM, Bajer A. Bartonella infections in three species of Microtus: prevalence and genetic diversity, vertical transmission and the effect of concurrent Babesia microti infection on its success. Parasites \& vectors. 2018 Dec 1;11(1):491. Evolution. 2012;12:4:335-7. 